\title{
Aerosole in Medizin und Veterinärmedizin
}

\author{
Vorsitzende: \\ P. Reinhold/Jena \\ H. Fehrenbach/Marburg
}

\section{Aerosols in Medicine and Veterinary Medicine}

5. Workshop des Arbeitskreises „Vergleichende Pathologie und Pathophysiologie des respiratorischen Systems“ der Fachgruppe Pathologie der Deutschen Veterinärmedizinischen Gesellschaft (DVG) in Zusammenarbeit mit der Sektion Pathophysiologie und Aerosolmedizin der Deutschen Gesellschaft für Pneumologie (DGP)

Mittwoch, 26. 3. 2003

\section{Editorial}

Inzwischen ist es schon fast zur Tradition geworden, dass der Arbeitskreis Vergleichende Pathologie und Pathophysiologie des respiratorischen Systems der Fachgruppe Pathologie der Deutschen Veterinärmedizinischen Gesellschaft zusammen mit der Sektion Pathophysiologie und Aerosolmedizin einen Workshop im Vorfeld der Jahrestagung der DGP abhält. Gemeinsame Interessen der experimentell arbeitenden Wissenschaftler aus beiden Gesellschaften haben den Workshop mit dem Ziel ins Leben gerufen, den Informationsaustausch zwischen Veterinär- und Humanmedizinern zu fördern. Dies spiegelt sich auch im Thema des diesjährigen Symposiums der DGP-Tagung in München Aerosole in Medizin und Veterinärmedizin wider. In dem Symposium wird ein weiter Bogen gespannt um Speziesunterschiede in der Aerosoldosimetrie unter verschiedenen Aspekten zu betrachten. Neben der Partikeldeposition stehen funktionelle sowie morphologische Gesichtspunkte der Partikelclearance und die inflammatorische Reaktion auf inhalierte Partikel im Vordergrund. Im zweiten Teil des Symposiums werden praktische Aspekte zum Design und zur Durchführung toxikologischer Inhalationsstudien sowie natürliche Staubbelastungen und die dadurch induzierten Pneumokoniosen beim Tier vorgestellt. Abschließend werden therapeutische Aspekte der Aerosolmedizin insbesondere unter dem Aspekt der Übertragung humanmedizinischer Ansätze in den veterinärmedizinischen Bereich diskutiert. Das breite Spektrum und der zusammenfassende Charakter des gemeinsam von der DVG und DGP getragenen Symposiums sollten ein Anreiz für jeden aerosolmedizinisch interessierten Mediziner, unabhängig von seinem Hintergrund, sein.

H. Schulz

Institutsangaben

GSF-Forschungszentrum für Umwelt und Gesundheit, Institut für Inhalationsbiologie

Korrespondenzadresse

PD Dr. med. H. Schulz · GSF-Institut für Inhalationsbiologie · Ingolstädter Landstraße 1 · 85758 Neuherberg

Bibliografie

Pneumologie 2003; 57: 166 - 177 @ Georg Thieme Verlag Stuttgart · New York · ISSN 0934-8387 
Aerosole in Medizin und Veterinärmedizin -

Partikeldeposition

Schulz $H$

GSF-Forschungszentrum für Umwelt und Gesundheit, Institut für Inhalationsbiologie, Neuherberg/München

Einleitung: Sowohl beim Menschen als auch bei verschiedenen Tierspezies werden Aerosole zu therapeutischen Zwecken eingesetzt. Die applizierte Dosis wird hierbei häufig auf die Menge des vernebelten Aerosols bezogen. De facto kann jedoch nur von denjenigen Partikeln eine therapeutische Wirkung erwartet werden, die nach Inhalation auf der Oberfläche der respiratorischen Schleimhäute deponiert werden. Die deponierte Aerosolmenge hängt von verschiedenen Faktoren ab; diese werden einerseits durch das Aerosol bestimmt - z. B. die Teilchengröße - und andererseits durch das Individuum - z. B. das Atemmuster. Die Totaldeposition charakterisiert die relative Abscheidung der pro Atemzug inhalierten Partikel im gesamten respiratorischen System, während Regionaldepositionen, wie extrathorakale, bronchiale oder alveoläre Deposition, die Partikelbelastungen in anatomisch definierten Regionen des Atemtraktes beschreiben. Ziel dieser kurzen Übersicht ist es, den Leser in die Depositionsmechanismen, in charakteristische Depositionkurven des Atemtraktes und in Speziesunterschiede einzuführen.

Depositionsmechanismen: Bei allen Spezies beobachtet man eine charakteristische Abhängigkeit der Totaldeposition vom Partikeldurchmesser (vgl. Abb.1). Die Totaldeposition weist bei Teilchen mit einem Durchmesser von 0,1 mm-1 mm ein Minimum auf und steigt sowohl bei kleineren als auch bei größeren Teilchen bis auf Werte von $100 \%$ an. Dieser Kurvenverlauf wird durch die Eigenbeweglichkeit der Partikel, d.h. ihre physikalischen Eigenschaften bestimmt [6]. Diese Eigenbeweglichkeit bedingt ein Abweichen der Partikel von der Gasströmung und führt damit zur Abscheidung im Atemtrakt. Die wichtigsten Depositionsmechanismen sind Impaktion, Sedimentation und Diffusion. Letztere ist für Partikel $<0,1 \mu \mathrm{m}$ entscheidend. Die diffusive Eigenbeweglichkeit der Partikel steigt umgekehrt proportional zur Wurzel des Partikeldurchmessers an. Für 0,1- bzw. 0,01- $\mu \mathrm{m}$-Partikel beträgt die mittlere diffusive Wegstrecke $39 \mu \mathrm{m} \mathrm{s}^{-1}$ bzw. $174 \mu \mathrm{m} \mathrm{s}^{-1}$. Diffusive Abscheidung spielt daher vor allem in kleinräumigen geometrischen Strukturen, wie sie im Alveolarbereich und in der Nase vorliegen, eine Rolle. Trotz ihrer geringen Masse unterliegen Partikel der Schwerkraft und sedimentieren. Die Sedimentationsstrecke ist proportional zum Quadrat des Teilchendurchmessers und führt bei 1-, 5-, bzw.
10- $\mu \mathrm{m}$-Partikeln zu einer Fallstrecke von $33 \mu \mathrm{m} \mathrm{s}^{-1}, 740 \mu \mathrm{m} \mathrm{s}^{-1}$ bzw. $2900 \mu \mathrm{m} \mathrm{s}^{-1}$. Lange Aufenthaltszeiten, z. B. beim Atemanhalten nach Inhalation, und kleinräumige geometrische Strukturen begünstigen damit die Deposition durch Sedimentation. Aufgrund ihrer Massenträgheit können Partikel plötzlichen Änderungen der Strömungsrichtung in den Atemwegen zum Teil nicht folgen und impaktieren so z.B. an der Bronchialwand. Bei einer Strömungsgeschwindigkeit von $1 \mathrm{~m} \mathrm{~s}^{-1}$ führt die Trägheit bei ei-

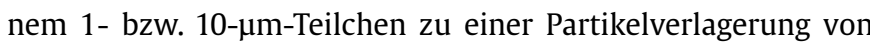
$3 \mu \mathrm{m}$ bzw. $300 \mu \mathrm{m}$. Forcierte Atmung mit hohen Strömungsgeschwindigkeiten begünstigt daher die Deposition von großen Teilchen im oberen Atemtrakt.

Total- und Regionaldeposition im Atemtrakt von Mensch und Ratte: Die vorgestellten Depositionsmechanismen machen die in Abb. 1 dargestellten Kurvenverläufe für Total- und Regionaldeposition während ruhiger Nasenatmung verständlich [1]. Beide Spezies zeigen den typischen Kurvenverlauf der Totaldeposition, mit einem Depositionsminimum für Partikel mit sehr geringer Eigenbeweglichkeit. Während sich im Speziesvergleich identische Totaldepositionswerte für Partikel $<0,1 \mu \mathrm{m}$ ergeben, beobachtet man für größere Partikel beim Menschen um bis zu 30\% höhere Depositionsraten. Partikel $>2 \mu \mathrm{m}$ werden durch Impaktion und Sedimentation vor allem extrapulmonal, d.h. in der Nase abgeschieden. Vergleichweise höhere Depositionswerte im extrapulmonalen Bereich des Menschen weisen auf die effektivere nasale Filtration für Teilchen dieses Größenbereiches hin. Die hohe Effektivität des Filters Nase zeigt sich zum einen daran, dass bei beiden Spezies mindestens $80 \%$ der inhalierten Partikel $>4 \mu \mathrm{m}$ extrapulmonal abgeschieden werden und dadurch nur wenige in die tieferen Atemwegsstrukturen gelangen. Zum anderen lässt sich die Effektivität beim Menschen durch den Vergleich von Mund- und Nasenatmung abschätzen (vgl. Abb.2). Diejenigen Partikel, die tiefer in den Atemtrakt eindringen können, werden dann zum Teil im tracheobronchialen Bereich deponiert. Hier sind die kleiner dimensionierten Atemwege der Ratte offensichtlich in ihrer Filterfunktion effizienter als die des Menschen - für 3- $\mu \mathrm{m}$-Partikel wird in der Ratte ein Depositionsmaximum von ca. $10 \%$ erreicht, beim Menschen werden nur $4 \%$ dieser Partikel abgeschieden. Im alveolären Bereich zeigen beide Spezies vergleichbare Kurvenverläufe mit einem Depositionsmaximum im Teilchengrößenbereich von $2-3 \mu \mathrm{m}$. Für ultrafeine Teilchen, d.h. Partikel $<0,1 \mu \mathrm{m}$, steigt die Depositionswahrscheinlichkeit mit abnehmendem Teilchendurchmesser im extrapulmonalen Bereich kontinuierlich an und erreicht bei der Ratte Werte von etwas über $40 \%$, beim Menschen von gut $30 \%$.
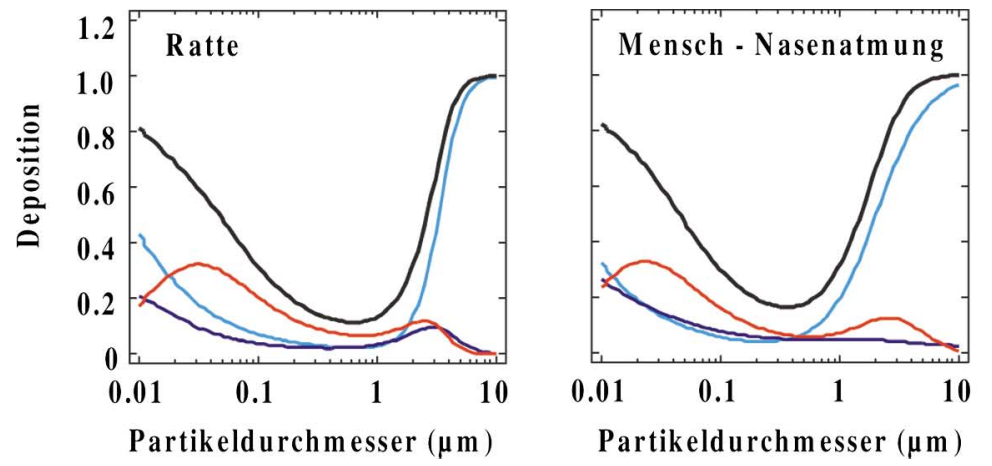

Abb. 1 Total- und Regionaldeposition als Funktion der Partikelgröße. Die für Mensch und Ratte dargestellten Werte gelten für Ruheatmung und beziehen sich auf nicht-hygroskopische Partikel mit einer Dichte von $1 \mathrm{~g} \mathrm{~cm}^{-3}$. 

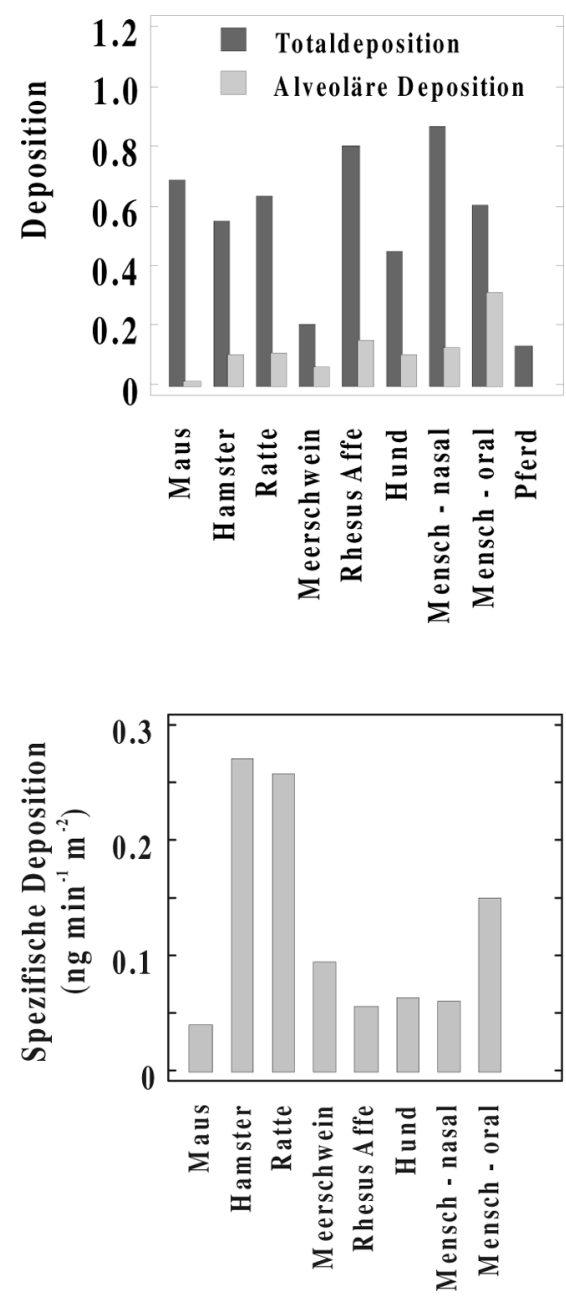

Abb. 2 Vergleich von Total- und alveolärer Deposition bei verschiedenen Spezies. Die Depositionswerte beziehen sich auf eine Teilchengröße von $3 \mu \mathrm{m}$ mit einer Dichte von $1 \mathrm{~g} \mathrm{~cm}^{-3}$ und gelten für Ruheatmungsbedingungen. Mensch - nasal: Nasenatmung, Mensch - oral: Mundatmung.

Abb. 3 Vergleich der alveolär deponierten Dosis in verschiedenen Spezies. Die pro Quadratmeter Alveolarfläche und Minute deponierte Dosis bezieht sich auf die Inhalation eines nicht-hygroskopischen 3- $\mu \mathrm{m}$-Aerosols mit einer Konzentration von $1 \mathrm{mg} \mathrm{m}^{-3}$. Berücksichtigt wurden typische Ruheatemmuster und die Oberfläche des Alveolarraumes der verschiedenen Spezies [3].

Für den tracheobronchialen Bereich beobachtet man eine ähnliche Abhängigkeit. Hier werden in der Ratte bis zu $20 \%$, beim Menschen bis zu 25\% deponiert. Da sehr kleine Partikel bereits effektiv extrapulmonal und tracheobronchial abgeschieden werden, erreicht die alveoläre Depositionskurve für ultrafeine Partikel ein Depositionsmaximun von nur etwa $30 \%$ bei $30 \mathrm{~nm}$ und fällt dann wieder ab.

Speziesunterschiede: Abb. 2 gibt eine Übersicht über die totale und alveoläre Deposition von 3- $\mu \mathrm{m}$-Partikeln unter Ruheatmungsbedingungen in 8 Spezies $[2,4,5,7]$. Für viele Vernebler ist dies ein typischer mittlerer Massendurchmesser. Bei Betrachtung der Abbildung fällt eine deutliche Variabilität zwischen den Spezies auf: für die Totaldeposition bei Nasenatmung liegen die Werte zwischen $13 \%$ und $86 \%$. Bei den meisten Spezies liegt die Totaldeposition jedoch bei Werten über $60 \%$. Relativ niedrige Werte zeigen Meerschwein, Pferd und Hund. Die alveoläre Deposition liegt in der Regel bei etwa 10\%. Dagegen deponiert nur 1\% der inhalierten 3- $\mu \mathrm{m}$-Partikel bei der Maus im alveolären Bereich, $15 \%$ bzw. $30 \%$ dagegen beim Rhesus-Affen bzw. beim Menschen während Mundatmung. Für das Pferd liegen keine Daten vor. Eine Korrelation zwischen Total- und alveolärer Deposition besteht nicht. Kritisch anzumerken ist, dass die hier zusammengestellten Depositionswerte aus verschiedenen Studien stammen und damit nicht auszuschließen ist, dass Differenzen zum Teil auf methodische Unterschiede zurückzuführen sind.

Dosimetrie - spezifische Deposition: Die bisher dargestellten Depositionswerte geben den relativen Partikelverlust aus der
Atemluft pro Atemzug an. Die vorgestellten Spezies weisen aber sehr unterschiedliche Atemmuster auf - z.B. atmet die Maus hochfrequent mit kleinem Atemzugvolumen und der Mensch langsam mit großem Atemzugvolumen. Hinzu kommt, dass sich die Gasaustauschfläche bei den Spezies deutlich unterscheidet bei der Maus stehen 0,1 $\mathrm{m}^{2}$ zur Verfügung, beim Menschen über $100 \mathrm{~m}^{2}$, auf die sich die deponierten Partikel verteilen können [3]. Beide Größen müssen zur Beurteilung der effektiv zur Wirkung kommenden Dosis berücksichtigt werden. Entsprechend zeigt Abb. 3 die pro Quadratmeter im Alveolarraum deponierte Masse eines 3- $\mu \mathrm{m}$-Aerosols mit einer Konzentration von $1 \mathrm{mg}$ $\mathrm{m}^{-3}$. Hier zeigt sich, dass Hamster und Ratte eine sehr hohe Dosis im Alveolarraum abscheiden. Für die übrigen Spezies ergibt sich ein relativ homogenes Bild. Die Abschätzung zeigt auch nochmals sehr schön, dass die alveoläre Dosis beim Menschen bei Nasenatmung deutlich niedriger als bei Mundatmung ist, da die

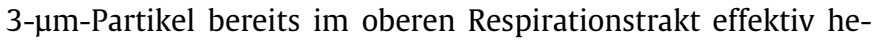
rausgefiltert werden.

\section{Literatur}

1 Casse FR, Freijer JI, Subramaniam R et al. Development of a model for human and rat airway particle deposition: implications for risk assessment. RIVM, Bilthoven NL, 2000: http://www.rivm.nl/bibliotheek/rapporten/650010018.pdf

2 Kreyling WG, Eder K, Erbe F et al. Particle deposition in the canine respiratory system. Ann Occup Hyg 1994; 38: 143 - 149

3 Parent RA. Comparative biology of the normal lung. Boca Raton: CRC Press, 1992

4 Raabe OG, Al-Bayati M, Teague SV et al. Regional deposition of inhaled monodisperse coarse and fine particles in small laboratory animals. Ann Occup Hyg 1988; 32: 53-63

5 Schlesinger RB. Comparative deposition of inhaled aerosols in experimental animals and humans: a review. J Toxicol Environ Health 1985; 15: $197-214$

6 Schulz H, Brand P, Heyder J. Particle deposition in the respiratory system. In: Gehr P, Heyder J (Hrsg). Particle-lung interactions. New York: Marcel Dekker, 2000: 229-290

7 Votion D, Ghafir Y, Munsters K et al. Aerosol deposition in equine lungs following ultrasonic nebulisation versus jet aerosol delivery system. Equine Vet J 1997; 29: 388 - 393

\section{Morphologische Grundlagen der Partikelclearance Morgenroth $K$ \\ Abteilung für Pathologie, Ruhr-Universität, Bochum}

Zur Abwehr partikulärer Bestandteile aus der Atemluft verfügt der Respirationstrakt über ein effektives System von Abwehrmechanismen. Erst bei einer Überschreitung seiner Kapazität oder einer Reduktion seiner Effektivität durch endogene oder exogene Faktoren ist mit krankhaften Veränderungen zu rechnen. Die Partikeldeposition im Lungengewebe führt zu einer proliferativen Entzündung mit protrahierter und irreversibler Vernarbung. Das Abwehrsystem besteht aus der Kombination unterschiedlicher zellulärer Leistungen, die in einem abgestimmten System zusammen wirken. Der Ablauf der Partikelelimination ist von der Größe und der Form der Teilchen abhängig.

Die histomorphologischen Grundlagen bilden dabei die Sekretion und der Sekrettransport im Bronchial- und Alveolarsystem in Interaktion mit den Alveolarmakrophagen.

Partikel über einem Durchmesser von $3 \mu \mathrm{m}$ werden durch die Sekretion und den Sekrettransport im Bronchialsystem entfernt. Durch Wirbelbildungen im Luftstrom der Bronchien sedimentie- 
ren die Partikel an der Bronchialwand. Sie bleiben auf der Oberfläche der Sekretschicht der Schleimhaut haften und werden mit dem Schleim durch den Zilienschlag nach außen in Richtung Larynx transportiert.

Die Effektivität dieses Mechanismus ist an bestimmte Eigenschaften des Bronchialsekretes gebunden, die einerseits eine Klebrigkeit zur Haftung der Partikel gewährleisten und andererseits durch ihre viskoelastischen Eigenschaften den Transport des Sekrets durch die Bewegung der Zilien ermöglichen müssen. Das Sekret stammt zu etwa $20 \%$ aus den Becherzellen des Oberflächenepithels und zu etwa $80 \%$ aus den in der subepithelialen Bindegewebszone angeordneten sero-mukösen Drüsen. Der Sekrettransport durch die Zilien des Bronchialepithels ist nur durch eine strenge Anordnung von zwei Komponenten auf der Epitheloberfläche möglich. Unmittelbar über dem Epithel liegt die dünnflüssige Solphase, in der die Zilien zum Schlag ausholen können. Darüber ist die Gelphase (die klebrige Komponente) angeordnet. Die Höhe der Solphase und die Herabsetzung der Klebrigkeit an der Basis der Gelphase wird durch Surfactant geregelt, der aus dem Alveolarbereich stammt [1].

Partikel unter einem Durchmesser von $3 \mu \mathrm{m}$ können direkt in den Alveolarraum gelangen. Auf der Oberfläche des Alveolarsystems liegt eine Flüssigkeitsschicht, über der eine Bedeckung mit Surfactant angeordnet ist. Der Surfactant wird bei der Ventilation in das Bronchialsystem verschoben. Auf der Schicht über dem Epithel haftende Partikel werden aus dem Alveolarraum in die Bronchiolen verlagert und hier über die sekretorischen Reinigungsmechanismen abtransportiert.

Die größte Bedeutung bei der Partikelelimination aus den Alveolen kommt den Makrophagen zu.

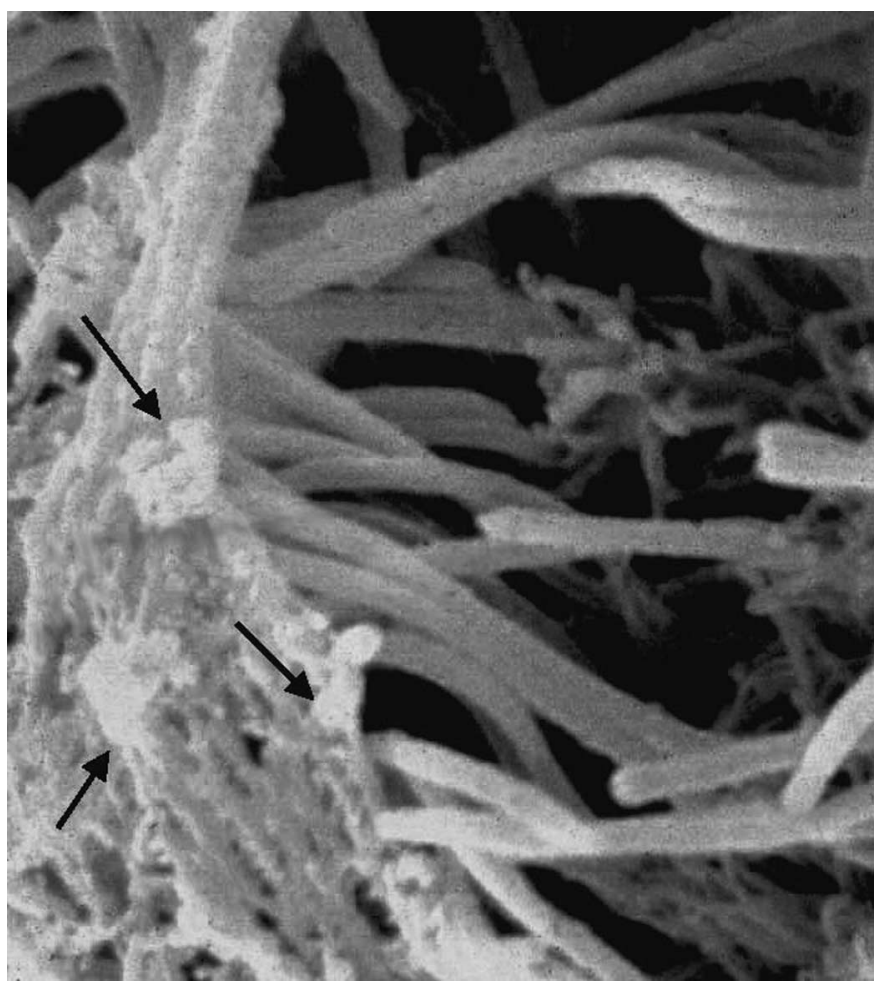

Abb. 1 Anordnung der Sekretschichten über dem Bronchialepithel. In der Umgebung der Zilien die dünnflüssige Solphase. Über den Zilien die Gelphase. Auf der Oberfläche der Sekretschicht anhaftende Partikel (Pfeile). Rasterelektronenmikroskopische Aufnahme, Vergrößerung: $18000 \times$

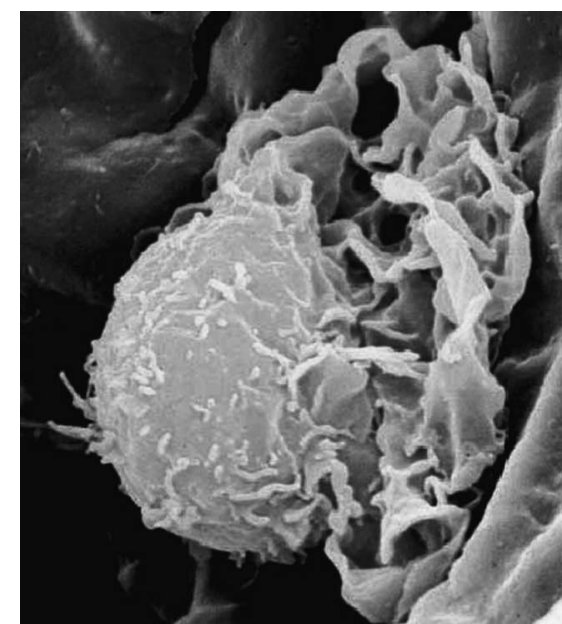

Abb. 2 Oberflächenstruktur eines Alveolarmakrophagen. Auf der Zelloberfläche eine dichte Anordnung von Microvilli und Microplicae. Rasterelektronenmikroskopische Aufnahme, Vergrößerung: $8200 \times$

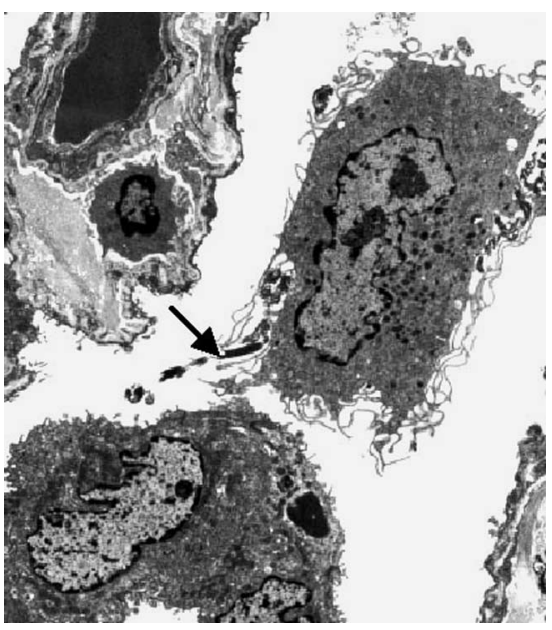

Abb. 3 In der Alveolarlichtung angeordneter Makrophage. Die Zelle hat über Microvilli mit einem Partikel Kontakt aufgenommen (Pfeil). Im Zytoplasma der Zelle eingeschlossene Partikel und viele Lysosomen. Vergrößerung: $7200 \times$

Sie wandern über das Interstitium durch die Interzellularspalten zwischen den Epithelzellen in die Lichtungen ein und können sich auf der Flüssigkeitsschicht und dem Surfactant frei bewegen. Die Oberflächenstruktur der Zellen ist durch dicht angeordnete Microvilli und Microplicae charakterisiert. Sie können lange Zellfortsätze bilden, mit denen sie mit Partikeln Kontakt aufnehmen. Durch die Kontaktaufnahme wird der Vorgang der Phagozytose in Gang gesetzt, über den die Partikel in das Zytoplasma der Zellen eingeschleust werden können. Es bildet sich dabei eine mundartige Einsenkung der Zellmembran, die tief in das Zytoplasma reicht. Die Zellmembran schließt sich über den Partikeln. In die Membran eingehüllt werden die aufgenommenen Partikel im Zytoplasma eingeschlossen.

Die morphologischen Befunde sprechen dafür, dass die Partikelaufnahme durch die Makrophagen durch den Surfactant vermittelt wird [2]. Die zunächst frei im Alveolarraum liegenden Partikel werden von Surfactant eingehüllt. In den Phagosomen sind neben den inkorporierten Partikeln membranöse Lipideinschlüsse nachweisbar, die diesem Surfactantanteil entsprechen. Die mit den Partikeln beladenen Zellen werden mit der Flüssigkeit bei der Ventilation in die Bronchiolen verlagert und mit dem Sekret der Bronchiolen und der Bronchien abtransportiert.

Für die Partikelclearance steht im Bronchial- und Alveolarsystem ein effektiver Schutzmechanismus zur Verfügung, in dem die Sekretion, der Sekrettransport in den Bronchien und die Leistung 
der Alveolarmakrophagen ineinander greifen. Das Ausmaß und die Folgen einer Partikelablagerung in der Lunge sind von der Größe der Partikel, der Partikelkonzentration in der Atemluft und der Leistungsfähigkeit des geschilderten Schutzmechanismus abhängig. Die Partikelinhalation hat besonders dann eine dauerhafte Wirkung mit einer Einschränkung der Lungenfunktion, wenn die Abwehrreaktionen durch exogene Faktoren wie das Zigarettenrauchen blockiert werden.

\section{Literatur \\ 1 Morgenroth K, Bolz J. Morphological feature of the interaction be- tween mucus and surfactant on the bronchial mucosa. Respiration 1985, 47: 225-231 \\ 2 Morgenroth K. Das Surfactantsystem der Lunge. Berlin: De Gruyter 1986}

Partilkelclearance aus dem Atemtrakt

Kreyling WG, Semmler M, Möller W

GSF - Forschungszentrum für Umwelt und Gesundheit, Institut für Inhalationsbiologie, Neuherberg/München

Nach der Deposition inhalierter Partikel auf den Epithelien des Atemtrakts sind die nächsten wichtigen dosimetrischen Determinanten die Retention der Partikel im Atemtrakt und ihre Clearance oder die ihrer Bestandteile aus dem Körper bzw. ihre Translokation in sekundäre Organe. Die Clearanceprozesse hängen generell sehr stark von den Materialeigenschaften der Partikel ab. Im folgenden werden lediglich Reinigungsprozesse für wasser- und lipidunlösliche Partikel diskutiert.

\section{Partikelclearance aus den Atemwegen}

Der wichtigste Clearanceprozess in den Luft-führenden Atemwegen ist der Mukoziliartransport, bei dem aufgrund der Aktivität des flimmernden Atemwegsepithels Mukus und darin suspendierte Partikel innerhalb von 1-2 Tagen zum Kehlkopf transportiert und abgeschluckt werden (Scheuch u. Mitarb. 1999). Darüber hinaus führt das Abhusten des Mukus mit den darin deponierten Partikeln ebenfalls zu ihrem Transport zum Kehlkopf. Dies ist ein wesentlicher Reinigungsvorgang bei Patienten mit immotilen Zilien (primäre ziliare Dyskinesie).

\section{Partikelclearance aus der Lungenperipherie}

Die Partikelclearance aus der Lungenperipherie, in der der Gasaustausch stattfindet, verläuft erheblich langsamer als die aus den Atemwegen, weil im Alveolarbereich kein Flimmerepithel und kein Mukus vorhanden ist. Die Kinetik der Langzeit-Retention kann sich über mehrere Jahre erstrecken, wie das anthrakotische Pigment in den Lungen von z.B. Bergarbeitern, Rauchern, aber auch von älteren Personen deutlich belegt. Die Partikel landen auf der epithelialen Flüssigkeitsschicht, die nur ca. $2 \mu \mathrm{m}$ dick ist. Sind die Partikel groß genug, so werden sie mit genügend molekularen Markern (Opsoninen) gekennzeichnet und innerhalb von wenigen Stunden durch Alveolarmakrophagen (AM) phagozytiert, die ihr weiteres Schicksal determinieren. Die Phagozytose durch AM hat ein Optimum bei Partikeln mit einer Grö-

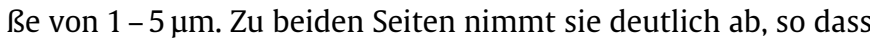
ultrafeine Partikel $<0,1 \mu \mathrm{m}$ und grob disperse Partikel $>10 \mu \mathrm{m}$ nur noch zu einem geringen Teil phagozytiert werden. Interessanterweise gelangen grob disperse Partikel nicht in die periphere Lunge, weil sie vorher in den oberen Atemwegen abgeschie- den werden. Dies trifft nicht für ultrafeine Partikel zu, die bis zu einer Größe von $20 \mathrm{~nm}(0,02 \mu \mathrm{m})$ vorzugsweise in den Alveolen deponiert werden; erst noch kleinere Partikel werden aufgrund ihrer noch höheren Diffusion auch in den oberen Atemwegen abgeschieden und erreichen in geringerem Maße in die Peripherie (Bair u. Mitarb., 1994).

Partikeltransportpfade aus der Lungenperipherie. Der wichtigste Clearancepfad unlöslicher Partikel aus der peripheren Lunge ist ihr Transport durch AM zunächst zum Beginn des Zilien-besetzten Flimmerepithels der terminalen Bronchioli und weiter zum Kehlkopf. Nach dem Verschlucken passieren sie den MagenDarm-Trakt und werden ausgeschieden, sofern sie nicht bei dieser Passage in ihre Bestandteile aufgeschlossen und resorbiert werden. Aufgrund von morphologischen Unterschieden in den respiratorischen Acini verschiedener Spezies variiert die Rate der AM, die täglich auf diese Weise zum Kehlkopf gelangen, um bis zu einer Größenordnung (Kreyling und Scheuch, 2000). So werden bei Nagern 1-3\% der AM Population pro Tag zum Kehlkopf verbracht, während nur $0,2-0,5 \%$ pro Tag der menschlichen AM auf diese Weise aus der Lunge eliminiert werden. Ähnliche Raten gelten auch für Affe und Hund. Dies bedeutet aber, dass die langzeitliche AM-abhängige Partikelclearance beim Menschen um eine Größenordnung langsamer verläuft als bei Nagern. Gleichzeitig nimmt der Partikelanteil mit der Zeit ab, der für diesen Clearancepfad zur Verfügung steht, weil die Partikel in andere Lungenkompartimente (z.B. Interstitium, Lympknoten) umgelagert und verkapselt werden. Diese Gründe führen dazu, dass beim Menschen insgesamt weniger als 50\% der unlöslichen Partikel aus der Lunge eliminiert werden. Der verbleibende Rest akkumuliert im Laufe der Zeit, wie das anthrakotische Pigment in den Lungen älterer Personen drastisch veranschaulicht. Hier sei aber auch erwähnt, dass die Lunge sehr effiziente Mechanismen zum Abbau, zur Lösung und zur Digestion von Fremdkörpern zur Verfügung hat. (Kreyling und Scheuch, 2000). In Langzeit-Clearanceuntersuchungen mit ferromagnetischen Testpartikeln aus dem Alveolarbereich konnte gezeigt werden, dass Zigarettenrauchen die Clearancekinetik maßgeblich verlangsamt. Bei älteren Rauchern stieg die Clearance-Halbwertsdauer von 130 Tagen auf über 450 Tage (Möller u. Mitarb., 2001, 2002). Diese Beeinflussung der Abwehrmechanismen kann mit ein auslösender Faktor der chronisch obstruktiven Bronchitis (COPD) sein. Eine Inhibition der Alveolarclearance ist auch bei Patienten mit interstitiellen Lungenerkrankungen (Sarkoidose, Fibrose) nachweisbar.

Wie bereits erwähnt, erfolgt die Phagozytose ultrafeiner Partikel durch AM nicht quantitativ, sondern diese Partikel werden u.a. auch von Epithelzellen aufgenommen bzw. finden Zutritt in das Interstitium. Die Vermutung liegt nahe, dass somit diese sehr kleinen Partikel mit einer anderen Rate in Richtung Kehlkopf eliminiert werden müssten. Hier gibt es noch keine Untersuchungen außer ersten Ergebnissen unserer eigenen Studien an einem Rattenmodell, in denen wir aber keinen Unterschied zur Clearancekinetik von größeren $(0,5-3 \mu \mathrm{m})$ Partikeln gefunden haben (Semmler u. Mitarb. 2003).

Der Partikeltransport in die tracheobronchialen Lymphknoten trägt nur geringfügig zur Elimination der Partikel aus dem Parenchym bei. Menschliche Daten zur Biokinetik sind äußerst rar. Wir haben in Hunden diesen Reinigungspfad quantifiziert: während der ersten Monate werden ca. 1 - 3\% der in der peripheren Lunge deponierten Partikel in die Lymphknoten transportiert und ver- 
bleiben dort. Der Transportvorgang unterliegt aber einer deutlichen biologischen Variabilität von Individuum zu Individuum (Kreyling und Scheuch, 2000). Diese Daten gelten allerdings nur für nicht-toxische Partikel.

Eine nennenswerte systemische Translokation von Partikeln $>0,5 \mu \mathrm{m}$ in die peripheren Blutgefäße konnte bisher unter physiologischen Bedingungen nicht nachgewiesen werden. Die systemische Translokation ultrafeiner Partikel ist umstritten; so suggerieren semi-quantitative Studien, dass bis zu 50\% der ultrafeinen elementaren Kohlenstoff-haltigen Partikel innerhalb von einem Tag systemisch transloziert werden und anschließend u. A. in der Leber akkumulieren (Nemmar u. Mitarb., 2001; Oberdörster u. Mitarb., 2002). Im Gegensatz dazu haben wir mit radioaktiv markierten, ultrafeinen Iridium-Partikeln in einem Rattenmodell lediglich eine translozierte Fraktion von ca. $1 \%$ bei Untersuchungen gefunden, bei denen eine quantitative Bilanz der Partikel im gesamten Körper und in der Ausscheidung durchgeführt wurde (Kreyling u. Mitarb., 2002). Interessanterweise haben wir die geringen Partikelfraktionen nicht nur in der Leber und Milz, sondern auch im Herzen und im Gehirn gefunden. Die widersprüchlichen Ergebnisse der anderen Autoren und unsere weisen darauf hin, dass die Transportmechanismen komplexer sind, als bisher angenommen. So könnten z. B. ultrafeine Partikel mit ähnlich großen Proteinen Komplexe eingehen, die über ihre weitere biokinetische Verteilung entscheiden und daher u.A. stark von den Oberflächenbeschaffenheiten der Partikel abhängen. Solche Partikel-Protein-Komplexe könnten wiederum die Funktion der Proteine verändern und damit zu adversen Gesundheitseffekten führen, wie sie zur Zeit den ultrafeinen Partikeln im Umweltaerosol aufgrund von epidemiologischen Studien zugeschrieben werden. Andererseits bieten solche ultrafeine, partikuläre Carriersysteme die Möglichkeit, in ihnen verpackte Medikamente gezielt über Membranbarrieren systemisch an ihre Wirkorte zu dirigieren. Diese Fragen sind Gegenstand intensiver laufender und neuer Untersuchungen.

\section{Literatur}

Bair WJ, Bailey MR, Cross FT et al. Human respiratory tract model for radiological protection. ICRP Publication 66. Annals of the ICRP 1994; 24: $1-3$

Kreyling WG, Scheuch G. Clearance of particles deposited in the lungs. In: Heyder J, Gehr P (Hrsg). Particle lung interactions. New York: Marcel Dekker 2000: 323 -376,

Kreyling WG, Semmler M, Erbe F et al. Ultrafine insoluble iridium particles are negligibly translocated from lung epithelium to extrapulmonary organs. J Tox Environ Health 2002; Vol. 65 (No. 20): 1513- 1530

Möller W, Barth W, Kohlhäufl M et al. Human alveolar long-term clearance of ferromagnetic iron-oxide microparticles in healthy and diseased subjects. Exp Lung Res 2001; 27: 547-568

Möller W, Kohlhäufl M, Häussinger K, Heyder J. Pneumologische Diagnostik mit Modellaerosolen, IV. Teil: Clearance von Aerosolpartikeln aus dem Alveolarbereich. Pneumologie 2002; 56: 503-510

Nemmar A, Hoet PH, Vanquickenborne B et al. Passage of inhaled particles into the blood circulation in humans. Circulation 2002; 105: $411-414$

Oberdörster G, Elder A, Gelein R et al. Extrapulmonary translocation of inhaled ultrafine carbon particles. J Toxicol Environ Health 2002; A 65 (20): $1531-1543$

Scheuch G, Kohlhäufl M, Sommerer K et al. Pneumologische Diagnostik mit Modellaerosolen, III. Teil: Clearance von Aerosolpartikeln aus den luftleitenden Atemwegen. Pneumologie 1999; 53: 329-336

Semmler M, Seitz J, Erbe F, Mayer P, Heyder J, Oberdoerster G, Kreyling WG. Long-term clearance kinetics of inhaled ultrafine insoluble iridium particles in the rat lung. Proceedings of the Fourth Colloquium on Particulate Air Pollution and Human Health, March 31 -April 2, 2003, Pittsburgh, Penn, USA
Speziesunterschiede in der inflammatorischen Reaktion der Lunge auf inhalierte Partikel

Krombach $F$

Institut für Chirurgische Forschung, Klinikum der Universität München-Großhadern, München

Eine Vielzahl an vergleichenden Untersuchungen hat gezeigt, dass jede Spezies ein ihr eigenes charakteristisches Depositions-, Retentions- und Clearancemuster aufweist und dass speziesabhängige Reaktions- und Schadensmuster auf inhaliertes Material beobachtet werden können. Insbesondere die in der Inhalationstoxikologie häufig verwendeten Versuchstierspezies, Ratten, Mäuse und Hamster, sind für ihre qualitativ unterschiedlichen pulmonalen Reaktionen auf inhalierte Partikel bekannt [9, 12, 18]. Als Ursache hierfür werden neben Speziesunterschieden in der Partikelclearance und -deposition speziesspezifische Variationen in der inflammatorischen Reaktion der Lunge auf zellulärer und molekularer Ebene diskutiert $[12,13,16]$. Speziesabhängige Variationen in Art und Ausmaß der inflammatorischen Reaktion auf inhalierte Partikel sind wahrscheinlich auch für die unterschiedliche Empfindlichkeit gegenüber dem „Overload“-Phänomen mit verantwortlich [14].

Seit langem wird vermutet, dass Speziesunterschiede in der Funktion der Alveolarmakrophagen (AM) einen Einfluss auf die biologische Reaktion des Gesamtorganismus auf inhalierte Noxen haben können [1]. AM haben die Aufgabe, inhalierte Mikroorganismen oder Partikel aufzunehmen, unschädlich zu machen und abzutransportieren sowie im Bedarfsfall weitere Entzündungszellen in den Alveolarraum zu rekrutieren. Zur Erfüllung dieser Aufgaben stehen diesem Zelltyp vielfältige Zellfunktionen zur Verfügung, wie z.B. die gerichtete Migration und die Phagozytose sowie die Freisetzung einer Vielzahl von Mediatoren, wie z. B. reaktive Sauerstoff- und Stickstoffspezies, Zytokine, chemotaktische Substanzen und Komplementfaktoren [4]. Vergleichende morphometrische Studien zeigten, dass menschliche AM einen deutlich größeren Durchmesser und damit ein vierbis fünffach größeres Zellvolumen aufweisen als AM von Ratte, Maus und Hamster [8, 11]. Diese Befunde legen nahe, dass sowohl die Anzahl als auch die Größe/Länge von Partikeln/Fasern, die durch AM vollständig phagozytiert werden können, durch die Zellgröße der AM bedingt sind. Hamster-AM weisen nach Exposition gegenüber Mineralfasern eine bessere vollständige Phagozytose der Fasern auf als Ratten-AM, während Ratten-AM im Vergleich mit Hamster-AM durch eine ausgeprägtere „frustrierte" Phagozytose und Sauerstoffradikalenproduktion charakterisiert sind [5]. Diese Ergebnisse werden durch ältere vergleichende Studien unterstützt, in denen beschrieben wurde, dass Hamster-AM eine höhere Phagozytoseaktivität aufwiesen als Rattenund Mäuse-AM [15, 17]. Interessanterweise scheint auch die oxidative Kapazität von Ratten- und Hamster-AM unterschiedlich $\mathrm{zu}$ sein [2]. Vergleichende In-vitro-Untersuchungen zeigten, dass es in Ratten-AM nach Stimulation mit Endotoxin und/oder Interferon-gamma zu einer Hochregulation der iNOS-mRNA-Expression und NO-Produktion kommt, während dies bei HamsterAM und menschlichen AM nicht der Fall ist $[3,10]$. Vergleichende In-vivo-Studien konnten kürzlich demonstrieren, dass in der Lunge der Ratte nach Exposition gegenüber Asbestfasern eine iNOS-mRNA- und -Proteinexpression sowie eine ausgeprägte inflammatorische Reaktion zu beobachten sind, während beim Hamster bei fehlender iNOS-Expression eine deutlich mildere in- 
flammatorische Reaktion induziert wird [6]. Untersuchungen an Asbest-exponierten iNOS-defizienten Mäusen weisen schließlich darauf hin, dass der iNOS-abhängigen NO-Produktion eine duale Rolle zukommt. Einerseits übt iNOS-abhängiges NO anti-inflammatorische Effekte über die Regulation der Produktion inflammatorischer Zytokine, wie z. B. TNF-alpha, aus, andererseits verstärkt iNOS-abhängiges NO den Partikel-induzierten pulmonalen Gewebeschaden, wahrscheinlich über die Bildung von Peroxynitrit [7].

Zusammenfassend deuten diese Befunde darauf hin, dass speziesabhängige Variationen in der Regulation der iNOS-Expression und NO-Produktion an den Speziesunterschieden in der pulmonalen Reaktion gegenüber inhalierten Partikeln mit beteiligt sind. In weiteren Studien sollten die den Speziesunterschieden in der inflammatorischen Reaktion der Lunge auf inhalierte Partikel zugrundeliegenden zellulären und molekularen Mechanismen systematisch untersucht werden, um eine bessere Grundlage für die Interpretation und Extrapolation von Ergebnissen aus tierexperimentellen In-vivo-Studien zu liefern.

\section{Literatur}

${ }^{1}$ Brain JD. Species differences in inhalation toxicology: variations in exposure-dose relationships and macrophage function. In: Dungworth $\mathrm{D}, \mathrm{Mohr} \mathrm{U}$ et al. (Hrsg). Inhalation Toxicology: The Design and Interpretation of Inhalation Studies and Their Use in Risk Assessment. Berlin: Springer Verlag, 1988: 1-23

${ }^{2}$ Dörger M, Allmeling A-M, Neuber A et al. Interspecies comparison of rat and hamster alveolar macrophage antioxidative and oxidative capacity. Environ Health Perspect 1997; 105 (Suppl 5): 1309-1312

${ }^{3}$ Dörger M, Jesch NK, Rieder G et al. Species differences in nitric oxide formation by rat and hamster alveolar macrophages in vitro. Am J Respir Cell Mol Biol 1997; 16: 413-420

${ }^{4}$ Dörger M, Krombach F. Interaction of alveolar macrophages with inhaled mineral particulates. J Aerosol Med 2000; 13: 369-380

${ }^{5}$ Dörger M, Münzing S, Allmeling A-M et al. Comparison of the phagocytic response of rat and hamster alveolar macrophages to man-made vitreous fibers in vitro. Hum Exp Toxicol 2000; 19: 635-640

${ }^{6}$ Dörger M, Allmeling A-M, Kiefmann R et al. Early inflammatory response to asbestos exposure in rat and hamster lungs: role of inducible nitric oxide synthase. Toxicol Appl Pharmacol 2002; 181: $93-105$

${ }^{7}$ Dörger M, Allmeling A-M, Kiefmann R et al. Dual role of inducible nitric oxide synthase in acute asbestos-induced lung injury. Free Radical Biol Med 2002; 33: 491 - 501

${ }^{8}$ Haley PJ, Muggenburg BA, Weissman DN, Bice DE. Comparative morphology and morphometry of alveolar macrophages from six species. Am J Anat 1991; 191: $401-407$

${ }^{9}$ Heinrich U, Muhle H, Takenaka S et al. Chronic effects on the respiratory tract of hamsters, mice and rats after long-term inhalation of high concentrations of filtered and unfiltered diesel engine emissions. J Appl Toxicol 1986; 6: 383-395

10 Jesch NK, Dörger M, Rieder G et al. Expression of inducible nitric oxide synthase and formation of nitric oxide by alveolar macrophages: an interspecies comparison. Environ Health Perspect 1997; 105 (Suppl 5): $1297-1300$

${ }^{11}$ Krombach F, Münzing S, Allmeling A-M et al. Cell size of alveolar macrophages: an interspecies comparison. Environ Health Perspect 1997; 105 (Suppl 5): 1261 - 1263

12 Mauderly JL. Usefulness of animal models for predicting human responses to long-term inhalation of particles. Chest 1996; 109 (3 Suppl): 65 S- 68 S

13 Maxim LD, McConnell EE. Interspecies comparisons of the toxicity of asbestos and synthetic vitreous fibers: a weight-of-the-evidence approach. Regul Toxicol Pharmacol 2001; 33: 319-342

14 Mossman BT. Mechanisms of action of low solubility particulates in overload-related lung pathology. Inhal Toxicol 1997; 12: 141-148

${ }^{15}$ Warheit DB, Hartsky MA, Stefaniak MS. Comparative physiology of rodent pulmonary macrophages: in vitro functional responses. J Appl Physiol 1988; 64: 1953-1959

${ }^{16}$ Warheit DB. Interspecies comparisons of lung responses to inhaled particles and gases. Crit Rev Toxicol 1989; 20: 1-29
${ }^{17}$ Warheit DB, Hartsky MA. Role of alveolar macrophage chemotaxis and phagocytosis in pulmonary clearance responses to inhaled particles: comparisons among rodent species. Microsc Res Techniq 1993; 26: $412-422$

${ }^{18}$ Warheit DB, Hartsky MA. Influences of gender, species, and strain differences in pulmonary toxicological assessments of inhaled particles and/or fibers. In: Dungworth DL, Mauderly JL, Oberdörster G (Hrsg). Toxic and carcinogenic effects of solid particles in the respiratory tract. Washington: ILSI Press, 1994: 253-265

\section{Untersuchungen zur Wirkung experimenteller}

Staub-Exposition

Durchfüihrung - Pathologie - Verteillungsmuster -

Bewertungskriterien

Rosenbruch $M$

Bayer AG - PH-PDP-T Pathologie Pharma-Forschungszentrum Wuppertal

Staubexposition als Ursache von Krankheiten spielt für den Menschen eine große Rolle. Nach Tagen, an denen erhöhte Staubkonzentrationen gemessen werden, kommt es in vielen Industrieländern zu einer Zunahme der Todesfälle [1]. Deshalb ist es für Fragestellungen der Arbeits- und Umweltmedizin, ebenso wie auch für die Entwicklung und Produktion von Arzneimitteln und anderen Wirkstoffen von großer Bedeutung, die Wirkung von Stäuben im Rahmen experimenteller Studien zu testen.

Bevor experimentelle Untersuchungen begonnen werden, müssen zuerst die physikalischen und chemischen Eigenschaften der zu testenden Stäube ermittelt werden. Darüber hinaus spielt die Korngrößenverteilung eine wesentliche Rolle. Erste experimentelle Untersuchungen sollten dann an In-vitro-Modellen durchgeführt werden. Dafür stehen zahlreiche Zellkultur-Systeme oder andere Modelle zur Verfügung, in denen erste Ergebnisse zur Wirkung der Testsubstanzen gewonnen werden können.

Für die experimentelle Untersuchung im Tiermodell bestehen grundsätzlich drei verschiedene Applikations- bzw. Expositionsmöglichkeiten: die intraperitoneale (ip) Injektion, die intratracheale (itr) Instillation und die Inhalation. Bei inhalativen Studien gibt es beispielsweise die „Nose-only“-Exposition, die „Headonly"-Exposition und die Ganzkörper-Exposition. Die Wahl der Testmethode erfolgt nach verschiedenen Kriterien, wie z.B. methodischem Aufwand, experimenteller Kapazität, oder dem Umfang der geplanten Auswertungen. Da Stäube beim Menschen hauptsächlich im Respirationstrakt wirken, sollten - wenn möglich - die intratracheale Instillation oder die Inhalation angewandt werden, damit die bei der natürlichen Exposition betroffenen Organe/Gewebe - besonders Nase, Kehlkopf, Bronchien und Lunge - für die pathomorphologische Auswertung zur Verfügung stehen. Wegen der z.T. sehr ausgeprägten Spezies-spezifischen Reaktionen ist - gerade bei Fragen der Kanzerogenität auch die Auswahl der Tierart von wesentlicher Bedeutung. Im Regelfall werden experimentelle Studien an Nagern, besonders Ratten, durchgeführt. Wenn es notwendig wird, Nichtnager-Spezies zur Untersuchung der Wirkung von Stäuben einzusetzen, kommt dies nur für Inhalationsstudien in Betracht. Im Regelfall werden hierfür Hunde genommen, da sie sich vergleichsweise gut für die Experimente trainieren lassen und da zahlreiche Vergleichsdaten vorliegen. Zur Durchführung von Inhalationsstu- 
Tab. 1 Applikations-/Expositions- und Auswertungsmöglichkeiten bei der experimentellen Untersuchung von Stäuben im Tiermodell

\begin{tabular}{|cll}
\hline Applikation/Exposition & Auswertungen & \\
\hline Intraperitoneal (ip) & In-life-Phase & Lungenfunktionsparameter \\
\hline Intratracheal (itr) & & Broncho-alveoläre Lavage (BAL) \\
\hline Inhalativ - Nose only & Post mortem & Sektion (inkl. Organgewichte) \\
- Head only & & $\begin{array}{l}\text { Qualitative Histopathologie } \\
\text { - Ganzkörper }\end{array}$ \\
& $\begin{array}{l}\text { (inkl. Spezialfärbungen, Immun- } \\
\text { histochemie, Elektronenmikro- } \\
\text { skopie, TEM, SEM) }\end{array}$ \\
& Morphometrie \\
\hline
\end{tabular}

dien bei Versuchstieren siehe die Übersichtsarbeiten von Heyder und Takenaka [2] sowie Pauluhn und Mohr [3].

Neben den Besonderheiten der In-life-Phase müssen auch für die postmortale Auswertung spezielle Maßnahmen getroffen werden: Anpassung des Sektionsganges, deutlich erweitertes Organbzw. Probenspektrum, besonders Festlegung der Fixierungsmethode und der Probenlokalisationen der Lunge. Bei der Festlegung der zu untersuchenden Organ-/Gewebs-Lokalisationen ist weiterhin zu bedenken, dass auch bei inhalativer Exposition Staub-induzierte Veränderungen an Organen außerhalb des Respirationstraktes auftreten können. Besonders in den LungenAssoziierten Lymphknoten kommt es sehr häufig - z.T. ohne eine Korrelation zu Lungenveränderungen - zu Staub-bedingten Läsionen [4].

Abhängig von der Größenverteilung und den übrigen Eigenschaften des zu testenden Staubes treten Veränderungen an unterschiedlichen Lokalisationen des Respirationstraktes auf. In den unterschiedlichen Anteilen der Nase sowie im Kehlkopf und im Bronchialbaum können beispielsweise Meta- und Hyperplasien der verschiedenen Epithelien auftreten, die meist nach einem angemessenen expositionsfreien Zeitraum (Recovery) reversibel sind. In der Lunge kann es - abhängig von den Eigenschaften der inhalierten Testsubstanz und der Expositionszeit z.B. nur zu einer Ansammlung phagozytierender Zellen, zu einer leichten entzündlichen Reaktion, oder zu einer ausgedehnten Fibrose kommen. Eine Reversibilität ist in den ersten beiden Fällen meist gegeben, bei einer manifesten Fibrose dagegen nicht.

Besonders bei der Abgrenzung und Bewertung geringgradiger Staub-induzierter Veränderungen ist der Vergleich zu der bei jedem Experiment unbedingt notwendigen Kontrollgruppe wichtig. Je nach Design und Fragestellung einer Studie kann es zweckmäßig bzw. notwendig sein, mehrere Kontrollgruppen vorzusehen (z.B. Negativ-Kontrollgruppe, Lösemittel-Kontrollgruppe, Positiv-Kontrollgruppe).

Während der Expositionsphase lassen sich besonders bei der Verwendung von Nichtnager-Spezies kontinuierlich Lungenfunktionsparameter bestimmen oder andere teils invasive, teils nicht-invasive Methoden anwenden, deren Ergebnisse Hinweise zur Bewertung der Staubwirkung geben. Besonders wertvolle Daten zu induzierten Prozessen in der Lunge erhält man durch die broncho-alveoläre Lavage (BAL), wobei auch die serielle Anwendung dieser Methode nicht zu langfristigen Veränderungen der Lungenstruktur führt [5].
Nach Abschluss der Expositionsphase sollten zur abschließenden Bewertung der Staubwirkung unbedingt pathomorphologische Untersuchungen durchgeführt werden. Nach einer genauen makroskopischen Beurteilung während der Sektion, einschließlich der Bestimmung der Organgewichte, folgt die qualitativ-histopathologische Untersuchung. Neben der routinemäßig durchzuführenden HE-Färbung können je nach Fragestellung und Befundspektrum alle weiteren Methoden der Pathologie angewandt werden (z. B. Spezialfärbungen, Immunhistochemie sowie Raster- und Transmissions-Elektronenmikroskopie). Gerade bei arbeits- und umweltmedizinischen Fragestellungen zur RisikoBewertung ist oft, zusätzlich zur qualitativen Auswertung und zur Korrelation mit den Ergebnissen der In-life-Phase, eine quantitative Untersuchung sinnvoll und notwendig. Dafür stehen zahlreiche morphometrische Methoden mit sehr unterschiedlichem methodischen Aufwand zur Verfügung. Je nach Fragestellung kann die Zählung bzw. Messung einzelner Läsionen (z.B. Tumoren) ausreichend sein, in anderen Fällen ist die aufwändigere Auswertung mit bildanalytischen Methoden notwendig.

Bei der Bewertung hinsichtlich möglicher Substanz-induzierter Veränderungen ist zwischen Spontanbefunden, Handling-induzierten Veränderungen und „wirklichen“ Substanz-Effekten zu unterscheiden.

Neben Vergleichen zu den Kontroll-Daten der zu bewertenden Studie ist es in diesem Zusammenhang oft unumgänglich, auf historische Kontroll-Daten zurück zu greifen.

Die abschließende Bewertung der Studien zur Wirkung einer experimentellen Staubexposition muss im Dialog aller beteiligten Disziplinen und unter Berücksichtigung aller Befunde erfolgen. Als Konsequenz kann es zur Festlegung von Grenzwerten oder zur Beschränkung der Anwendung einer Substanz oder eines Wirkstoffes kommen.

\section{Literatur}

1 Die wichtigsten Entwicklungen der Arbeitsmedizin: Deutsches Ärzteblatt 2002; 99 (27): C1477

2 Heyder J, Takenaka S. Long-term canine exposure studies with ambient air pollutants. Eur Resp J 1996: $571-584$

3 Pauluhn J, Mohr U. Inhalation studies in laboratory animals - current concepts and alternatives. Toxicol Pathol 2000; 28: 734-753

4 Rosenbruch M, Idel H, Friedrichs $\mathrm{KH}$ et al. Vergleichende Untersuchungen zur Wirkung von Quarzglas und Quarz DQ-12 im Inhalationsversuch bei Ratten. Zbl Hyg 1990; 189: 419-440

5 Krombach F, Fiehl E, Burkhardt D, Rienmüller R, König G, AdelmannGrill BC, Idel H, Rosenbruch M. Short-term and long term effects of serial bronchoalveolar lavages in a non-human primate model. Am J Respir Crit Care Med 1994; 150: 153 - 158

\section{Natuirliche Staubbelastung und Pneumokoniosen beim Tier \\ Theegarten $D$ \\ Abteilung für Pathologie, Ruhr-Universität, Bochum}

Natürlich auftretende pulmonale Staubablagerungen und Pneumokoniosen sind auch beim Tier möglich. Diese Veränderungen werden anhand eigener Untersuchungen und der Ergebnisse der Literatur im folgenden weitergehend beschrieben.

\section{Natürliche Staubbelastung beim Tier}

Bei eigenen Untersuchungen an Lungen von 500 konventionell gehaltenen Altsauen waren nur gelegentlich minimale Staubablagerungen zu sehen. Diese lagen teilweise innerhalb von Alveo- 


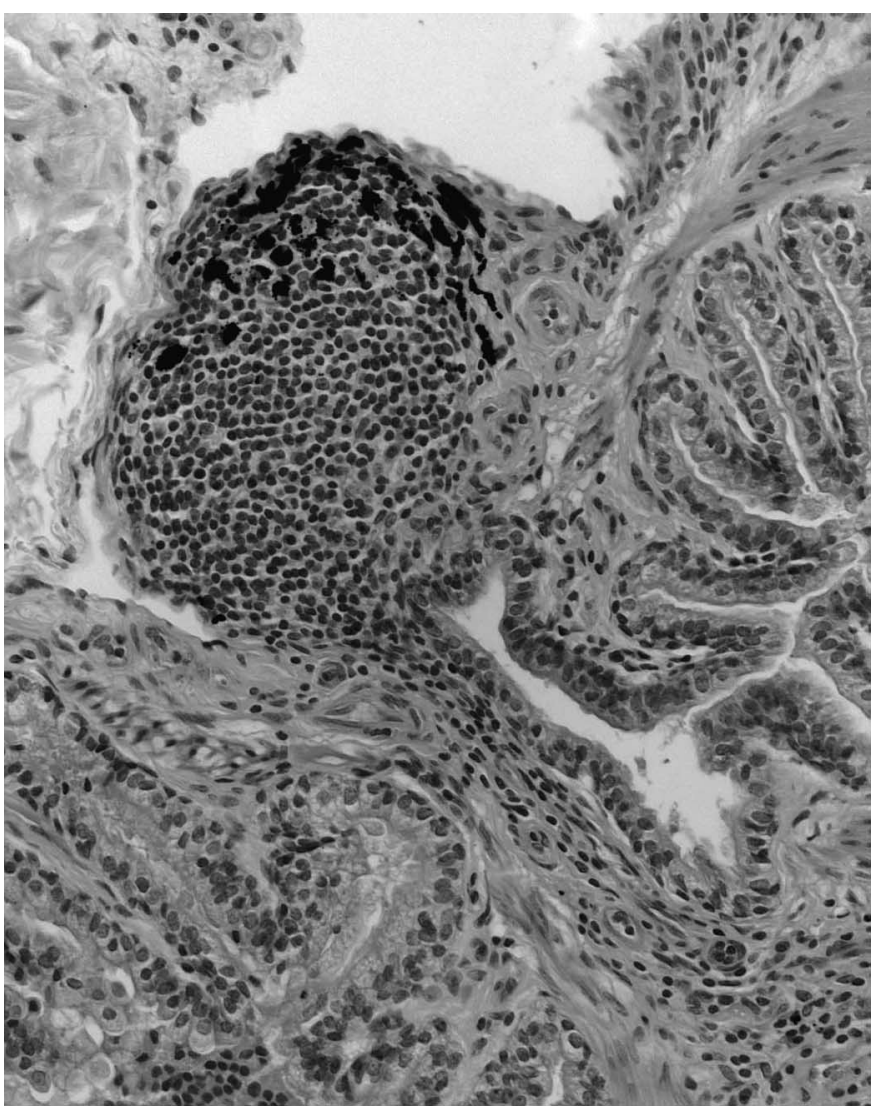

Abb. 1 a In den Lungen von Altsauen werden nur ganz gelegentlich Staubablagerungen innerhalb des Bronchus-assoziierten lymphatischen Systems nachgewiesen (Berliner-Blau-Färbung, 200×).

larmakrophagen und teils im Bereich des Bronchus-assoziierten lymphatischen Systems (Abb.1a). Bei Pferden konnten demgegenüber regelmäßig zumindest geringe peribronchioläre und perivasale Ablagerungen gefunden werden (Abb.1 b). Anthrakosilikosen wurden aber selbst beim Einsatz von Pferden in deutschen Steinkohlenzechen nicht beobachtet [14]. Eine Anthrakose der Lunge bzw. der Lymphknoten konnte weiterhin in 3,85\% von 700 unselektierten Rindern in der Türkei gefunden werden [8].

\section{Umwelt-assoziierte Pneumokoniosen beim Tier}

Bei der Untersuchung von 134 Kamelen in Somalia fanden sich in 16\% eine Staubfibrose und in 4,5\% Silikose-Knötchen, teilweise auch mit perifokalem Emphysem [6]. Bei über 40 Jahre alten menschlichen Bewohnern der chinesischen Wüste war bei 21\% eine Silikose feststellbar, wobei die Veränderungen mit dem Alter zunahmen [15]. Auch in den Lungen von Säugetieren und Vögeln aus dem Zoo von San Diego (Kalifornien/USA) konnten der Lage in einer Halbwüstenregion entsprechend komplexe Silikate aus der Umgebungsluft nachgewiesen werden, die in Abhängigkeit vom Alter, der Länge des Aufenthaltes im Zoo und Speziesabhängigen Faktoren auftraten [2]. Bei Pferden kann eine Silikose ebenfalls infolge von besonderen geologischen Bedingungen vorkommen. In einer Studie über 9 Tiere in Kalifornien/USA kamen diese von der Monterey-Carmel-Halbinsel, die aus marinen, Diatomeen-reichen Sedimenten aus dem Miozän aufgebaut ist. Bei den betroffenen Tieren entwickelte sich eine progrediente Dyspnoe mit Gewichtsverlust und silikotischen Knötchen, ähnlich wie bei der humanen Form [1,12]. Bei Husarenaffen, die vor-

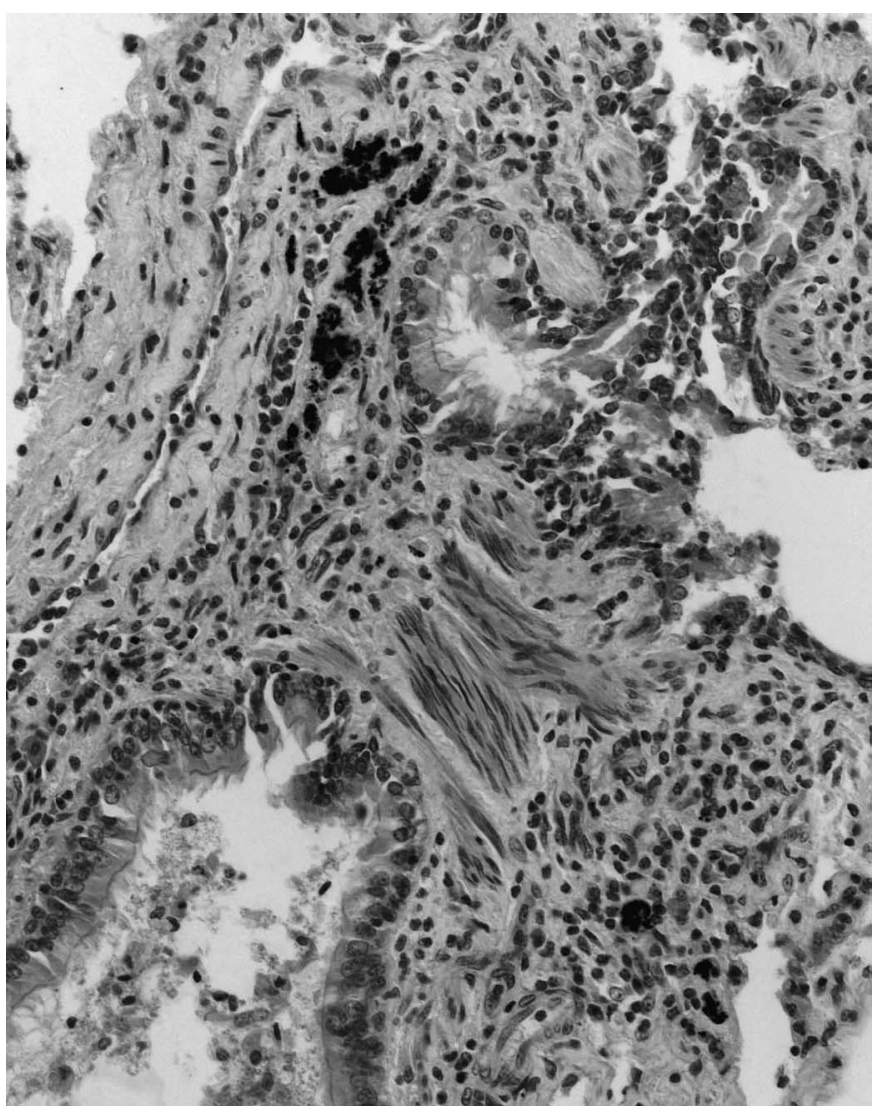

Abb. 1 b Bei Pferden finden sich in der Regel geringe peribronchioläre und perivasale Staubablagerungen (Berliner-Blau-Färbung, 200×).

nehmlich Bodenbewohner in Steppenregionen sind, wurden ebenfalls Pneumokoniosen mit Nachweis von Diatomeen gefunden [4].

\section{Haltungs-assoziierte Pneumokoniosen beim Tier}

Bei 4 einjährigen Schweinen, die in der Nähe von Zementwerken und Kalksteinbrüchen gehalten wurden, konnte eine Pneumokoniose nachgewiesen werden, die der gegebenen Exposition entsprach und mit der Energie-dispersiven Röntgenmikroanalyse dieser zugeordnet werden konnte [10]. Bei der Untersuchung von Hühnern aus einer Haltung mit ähnlicher Exposition waren bei 10 je 3-4 Jahre alten Tieren ebenfalls deutliche Pneumokoniosen zu finden, dies galt auch für 3 je 3 Jahre alte Tiere aus einer Haltung in der Nähe einer Ziegelei [9]. Bei 18 in Gefangenschaft gehaltenen Kiwis, die histologisch untersucht wurden, konnten bei 12 Tieren Pneumokoniosen beobachtet werden. Bei einem Teil der Vögel traten eine allgemeine Depression und ein Appetitverlust auf. Als Ursachen wurde eine Haltung in staubigen und trockenen Volieren in Verbindung mit der anatomischen Lage der Nasenlöcher und der besonderen Kopfhaltung bei der Nahrungssuche angenommen [13]. Ähnliche haltungsbedingte Pneumokoniosen wurden auch bei Ringfasanen [5] und bei Meerschweinchen gefunden [7].

Weiterhin wurde über eine extrapulmonale Silikose bei 2 alten Wasserbüffeln [11] und auch über eine Pneumokoniose bei 2 Hunden berichtet [3], wobei die Ursache jedoch unklar blieb. 


\section{Schlussfolgerungen}

Die Entwicklung pulmonaler Staubablagerungen ist auch beim Tier eine Frage der Exposition und derer Dauer auf der einen sowie der mukoziliären Clearance auf der anderen Seite. Eine Expositionszeit von mehreren Jahren wird aufgrund der Haltungsdauer nur von einem Teil der Nutztiere erreicht. Die beim Tier vorkommenden unterschiedlich starken Staubablagerungen und Pneumokoniosen sind eine Folge von natürlichen oder exogen bedingten Umweltbedingungen und in ihrer Ausprägung abhängig von der Spezies und dem Alter der Tiere. Zur Diagnostik trägt außer der sorgfältigen Haltungsanamnese und der Routinehistologie die Röntgenmikroanalyse im Rasterelektronenmikroskop bei.

\section{Literatur}

${ }^{1}$ Berry CR, O'Brian TR, Madigan JE et al. Thoracic radiographic features of silicosis in 19 horses. J Vet Intern Med 1991; 5: 248-256

${ }^{2}$ Brambilla C, Abraham J, Brambilla E et al. Comparative pathology of silicate pneumoconiosis. Am J Path 1979; 96: 149-169

${ }^{3}$ Canfield PJ, Rothwell TLW, Papadimitriou JM et al. Siliceous pneumoconiosis in two dogs. J Comp Path 1989; 100: 199-202

${ }^{4}$ Dayan AD, Morgan RJI, Trefty BT et al. Naturally occuring diatomaceous pneumoconiosis in sub-human primates. J Comp Path 1978; 88: $321-325$

${ }^{5}$ Evans MG, Slocombe RF, Schwartz LD. Pulmonary silicosis in captive ring-necked pheasants: Definitive diagnosis by electron probe $\mathrm{X}$-ray microanalysis. Vet Path 1988; 25: 239-241

${ }^{6}$ Hansen HJ, Jama FM, Nilsson C et al. Silicate pneumoconiosis in camels. J Vet Med A 1989; 36: 789-796

${ }^{7}$ Muto T. Spontaneous organic dust pneumoconiosis in guinea pigs. Jap J Vet Sci 1984; 46: 925-927

8 Özcan K, Beytut E. Pathological investigations on anthracosis in cattle. Vet Record 2001; 149: 90-92

${ }^{9}$ Roperto F, Borzacchiello G, Ungaro R et al. Silicate pneumoconiosis in hens. J Comp Path 2000; 122: 249-254

${ }^{10}$ Roperto F, Damiano S, de Vico G et al. Silicate pneumoconiosis in pigs: Optical and scannino electron microscopical investigations with $\mathrm{x}$-ray microanalysis. J Comp Path 1994; 110: 227-236

${ }^{11}$ Roperto F, Troncone A, Tranquillo A et al. Extrapulmonary silicosis in two water buffaloes. J Comp Path 1995; 112: 97-103

12 Schwartz LW, Knight HD, Whittig LD et al. Silicate pneumoconiosis and pulmonary fibrosis in horses from the Monterey-Carmel peninsula. Chest 1981; 80: $82 \mathrm{~s}-85 \mathrm{~s}$

${ }^{13}$ Smith BL, Poole WSH, Martinovich D. Pneumoconiosis in the captive New Zealand kiwi. Vet Path 1973; 10: 94-101

14 Theegarten D. Das Grubenpferd im rheinisch-westfälischen Bergrevier - ein historischer Rückblick. Der Anschnitt 2002; 54: 165 - 169

${ }^{15} \mathrm{Xu}$ X-Z, Cai X-G, Men X-S et al. A study of siliceous pneumoconiosis in a desert area of Sunan County, Gansu Province, China. Biomed Environment Sci 1993; 6: 217-222

\section{Effizienz von therapeutischen Aerosol-Applikationen bei Heim- und Nutztieren \\ Kirschvink $N$ \\ Department for Functional Sciences B41, Faculty of Veterinary Medicine, University of Liège, Belgium}

Aerosole werden beim Heim- und Nutztier vor Allem bei Katze, Hund, Pferd und in geringerem Maße beim Rind zu therapeutischen Zwecken appliziert. Die Hauptindikationen sind bei der Katze das so genannte Katzenasthma oder die chronische Bronchitis (Padrid, 2000), beim Hund die chronische eosinophile Bronchitis (Clercx, 1997), beim Pferd Dämpfigkeit (Heuallergie) sowie chronische, nicht-allergische Entzündungen der unteren Atemwege (Duvivier u. Mitarb., 1997) und beim Rind der so ge- nannte „bovine respiratorische Komplex (bovine respiratory complex)“, ein akutes Atemwegs- und Lungensyndrom bakteriellen und viralen Ursprungs (Lekeux, 1996).

Da von Seiten des Tieres keine Kontrolle der Atmung während der Applikation von Aerosolen vorhanden ist, werden diese durch passive Atmung inhaliert. Dabei spielen das Verhalten des Patienten, anatomische und größenbedingte Besonderheiten des Atmungsapparates sowie pathologisch bedingte Veränderungen des Atmungsverhaltens eine Rolle sowohl für die Wahl des Inhalators (Ultraschall oder Druckluft, dry powder inhaler [dpi], pressurized metered dose inhaler [pmdi]) als auch für die Art der Verabreichung der Aerosole (Inhalationskammer, Masken mit oder ohne Spacer).

Aufgrund ihrer Größe können Aerosole bei Katzen und den meisten Hunden anhand von Inhalationskammern aus Plexiglas appliziert werden. Das Aerosol wird entweder anhand eines Ultraschall-Nebulizers oder anhand eines Druckluft-Nebulizers generiert, wobei letzterer bei geräuschempfindlichen Katzen nicht immer toleriert wird. Nebulizer aus der Humanmedizin können für diese Zwecke genutzt werden. Diese Anwendungsform ist jedoch hauptsächlich den Tierkliniken vorbehalten und hat den Nachteil, dass ein großer Teil des Aerosols vom Patienten über Lecken aufgenommen wird sowie in manchen Fällen zu Reizungen der Konjunktiva führen kann. Als Alternative können kleine Masken, die Nasen- und Maulpartie des Patienten umfassen und mit einem Spacer geringen Volumens $(\sim 150 \mathrm{ml})$ und einer inspiratorischen Einwege-Klappe geringen Widerstandes versehen sind, zur Applikation von Aerosolen aus pmdi benutzt werden. Hierbei ist eine gewisse Toleranz von Seiten des Patienten nötig, da der Spacer innerhalb kurzer Zeit geleert werden sollte und das Tier gleichmäßig und ruhig atmen muss. Die Nutzung eines pmdi erlaubt den Einsatz verschiedener Moleküle (Sympathikomimetika, Anticholinergika, Kortikosteroide) aus der Humanmedizin. Allerdings sind bei Hund und Katze nur wenige wissenschaftlich belegte Inhalationstherapien beschrieben, was auf den Mangel an klinisch durchführbaren Lungenfunktionstests zurückzuführen ist. Die seit Kurzem beschriebene Ganzkörperplethysmographie bei der Katze (Hoffman u. Mitarb., 1999) sollte jedoch in Zukunft eine breitere Anwendung finden und zur Erfassung der Effizienz von Aerosolen bei Katze und Hund beitragen. Beim Pferd ist die Applikation von Aerosolen anhand mehrerer Methoden möglich, da sein großer inspiratorischer maximaler Luftfluss genutzt werden kann (peak inspiratory flow: 120 bis 180 l/min). Beim Pferd ist aufgrund der Anatomie im Pharynxbereich keine Mundatmung möglich, eine Aerosolapplikation kann allein durch die nasale Inhalation erfolgen. Durch die Größe bzw. Länge und Form der Nasenhöhlen wird ein großer Teil des Aerosols gefiltert (Partikel, deren Durchmesser über $5 \mu \mathrm{m}$ liegt), weshalb die meisten Partikel einen Durchmesser zwischen 1 und $5 \mu \mathrm{m}$ haben sollten. Die in der Humanmedizin gebrauchten Druckluft-Nebulizer erzeugen meistens nur ungenügend Partikel von entsprechendem Durchmesser sowie einen unzureichenden Luftfluss. Für Pferde adaptierte Druckluft-Nebulizer erzeugen einen Druck von 6 bar, wobei ihre Effizienz besser ist als die eines Ultraschall-Nebulizers (Votion u. Mitarb., 1997), jedoch mit einem hohen Geräuschpegel des Kompressors einhergeht. Beim Gebrauch von Druckluft- oder Ultraschall-Nebulizern wird das Aerosol meistens anhand einer offenen Maske appliziert, wobei ein Teil des Aerosols in das direkte Umfeld des Pferdes entweicht. Beim Gebrauch von fest am Kopf anliegenden Masken, die mit 
inspiratorischen sowie exspiratorischen Dichtungsklappen versehen sind, können Aerosole anhand von pmdi oder dpi über einen Spacer oder direkt in die Maske verabreicht werden. Bei dieser Art der Verabreichung spielen die Qualität der Maske sowie das Atemverhalten des Pferdes eine wichtige Rolle (Atemfrequenz sowie Tidalvolumen), da das Aerosol durch die Inspiration des Patienten in die Atemwege gelangt. Weitere Aerosolapplikationen sind durch den Gebrauch von pmdi anhand von einem Nüstern-angepassten Spacer mit inspiratorischer Dichtungsklappe oder einem in einen Nüstern einzuführenden „Revolver“ möglich und leicht anwendbar. Aerosolapplikationen kommen vor Allem beim dämpfigen Pferd zum Einsatz, vorzugsweise werden Sympathikomimetika, Anticholinergika sowie Kortikosteroide verabreicht. Die klinische Effizienz der verschiedenen Therapien ist beim Pferd anhand von Lungenfunktionstests und Lungenszintigraphie besser erfasst als beim Kleintier (zur Übersicht: Duvivier u. Mitarb., 1997).

Beim Rind ist der Gebrauch von Druckluft-Nebulizern und einer offenen Maske zur Verabreichung von Sympatikomimetika sowie Anticholinergika beschrieben (Genicot u. Mitarb., 1995). Aufgrund des epidemischen Charakters des bovinen respiratorischen Komplexes, des notwendigen Zeitaufwandes einer Behandlung und einer vergleichbaren Effizienz parenteral verabreichter Moleküle ist die Aerosoltherapie beim Rind jedoch wenig verbreitet.

\section{Literatur}

Clercx C. Eosinophilic bronchopulmonary disease in dogs: clinical characteristics, treatment and follow-up. Proceedings of the $15^{\text {th }}$ Veterinary Respiratory Symposium, October 24-25, 1997, Liège, Belgium, pp 37-41

Duvivier DH, Votion D, Vandenput $\mathrm{S}$ et al. Aerosol therapy in the equine species. Vet J 1997; 154: 189-202

Genicot B, Close R, Lindsey JK, Lekeux P. Pulmonary function changes induced by three regimens of bronchodilating agents in calves with acute respiratory distress syndrome. Vet Rec 1995; 137: 183-186

Hoffman AM, Dhupa N, Cimetti L. Airway reactivity measured by barometric whole-body plethysmography in healthy cats. Am J Vet Res 1999; 60: 1487 - 1492

Lekeux P. Le complexe respiratoire bovin. Annales de Médecine Vétérinaire 1996; $140: 101$ - 105

Padrid P. Feline asthma. Diagnosis and treatment. Vet Clin North AM 2000; 30: 1279-1293

Votion D, Ghafir Y, Munsters K et al. Aerosol deposition in equine lungs following ultrasonic nebulisations versus jet aerosol delivery system. Equine Vet J 1997; 29: 388 - 393

\section{Aerosoltherapie in der Pferdepraxis \\ - Indilkationen, praktische Durchführung, Probleme \\ Fey $\mathrm{K}$ \\ Medizinische und Gerichtliche Veterinärklinik der \\ Justus-Liebig-Universität Gießen \\ Professur für Innere Krankheiten der Pferde, \\ Gießen}

Indikationen: Salinische Aerosole ohne Zusatz verschreibungspflichtiger Substanzen sind bei allen entzündlichen Veränderungen der oberen und unteren Atemwege indiziert. Beim Tier bietet sich die Arzneigabe per Aerosol insbesondere bei chronischen Erkrankungen der Atemwege an, die keine häufige tierärztliche Überwachung erfordern. Die Tierbesitzer sollten die Behandlungen nach Anweisung durch den Tierarzt zu Hause bzw. im Stall selbst durchführen können. Von Vorteil sind weiterhin die im Vergleich zur enteralen oder parenteralen Behandlung häufig mögliche Dosisreduktion, eine bessere Compliance seitens Besitzer und/oder Patient sowie die möglicherweise bessere lokale Verträglichkeit des Arzneimittels bei inhalativer Gabe.

Häufigste Indikation einer Aerosoltherapie beim Pferd ist in Deutschland die chronische Bronchitis bzw. Bronchiolitis, gefolgt von pharyngealen lymphoiden Hyperplasien. Kortikosteroide und Bronchodilatatoren kommen am ehesten zum Einsatz. Bei bakteriellen Bronchopneumonien kann die inhalative Verabreichung von Antibiotika indiziert sein, aufgrund rechtlicher Probleme und mangelnder Datenlage zur Wirksamkeit wird dies aber wohl für absehbare Zeit Einzelfällen vorbehalten bleiben. Die inhalative Gabe von Arzneimitteln mit dem Ziel, systemische Wirkungen zu erreichen, spielt beim Pferd nach Kenntnis der Autorin bislang keine Rolle.

Praktische Durchführung: Am einfachsten für Pferd und Betreuer ist das Verabreichen von Aerosolen in einem komplett vernebelbaren Raum. Offensichtlich bietet sich dies nur für rein salinische Aerosole an. Von großem Vorteil dabei ist, dass sich das Tier frei und unbeaufsichtigt in der Box bewegen kann. Nachteilig ist allerdings, dass sich die Feuchtigkeit auf Boxenwänden und Haarkleid niederschlägt, was Hautpilzinfektionen Vorschub leisten kann. Der bauliche Aufwand und der Bedarf an inhalativer Verabreichung von Arzneimitteln lassen die möglichst direkte Gabe medikierter Aerosole in die Nüstern als praktikabler erscheinen. Grundsätzlich stehen für die Bereitstellung eines an der Pferdelunge wirksamen Aerosols die gleichen Möglichkeiten zur Verfügung wie in der Humanmedizin:

- Herstellung des Aerosols in Patientennähe mittels Vernebler (betrieben per Druckluft oder Ultraschall) sowie

- Verwendung von Metered Dose Inhalern (MDI). Dry Powder Formulierungen werden nach Kenntnis der Autorin bislang lediglich experimentell erprobt.

Wird ein Vernebler in Patientennähe, d. h. im Stall, eingesetzt, so liegt der entscheidende Vorteil in der Möglichkeit, die Behandlung über längere Zeit in Ruheatmung durchzuführen. Auch ist die inhalative Applikation isotoner oder leicht hypertoner Lösungen zur Mukosabefeuchtung und Sekretolyse möglich. Da in der Pferdemedizin die gleichen Tröpfchengrößen gefordert werden wie in der Humanmedizin, kommen als Vernebler Druckluftund Ultraschallvernebler der gleichen Hersteller zum Einsatz. Ist im Stall ein Kompressor vorhanden, so handelt es sich bei der Tröpfchenherstellung durch Druckluft um die bei Weitem preisgünstigste Methode, ein Pferdelungen-wirksames Aerosol herzustellen. Einige Pferde werden den in ihrer Nähe entstehenden Lärm allerdings nicht tolerieren. Fast lautlos und damit wenig irritierend für die Vierbeiner arbeiten Ultraschallvernebler, die bei der zu fordernden Leistungsfähigkeit in Tröpfchengröße und Output in der Anschaffung allerdings deutlich teurer sind. Beide Dauervernebler-Typen haben aus praktischen Gesichtspunkten allerdings folgende Nachteile: sie erfordern für die Verabreichung des von ihnen produzierten Aerosols nicht nur eine Maske (die die meisten Tiere gut tolerieren, wenn sie auch an Halfter und Trense gewöhnt sind), sondern auch Schlauchanschluss und die nicht in jedem Stall unproblematische Stromzufuhr. Eine Gewöhnung besonders an die zuführenden Schläuche kann im Einzelfall durchaus schwierig sein. Da man den Bewegungsradius von Kopf und Hals beim wachen Pferd auch durch Anbindung nicht völlig einschränken kann, ist eine Mindestlänge 
der Zuführung von $80 \mathrm{~cm}$ angezeigt. Ein Ultraschallvernebler muss also in der Nähe aufgestellt werden und längere Schläuche schützen zwar das Gerät bei unvorhergesehenen Bewegungen, führen aber auch zu höheren Aerosolverlusten.

Zum Zweiten ist das Atemzugvolumen eines typischen $600 \mathrm{~kg}$ Patienten von ca. 61 (und das Atemminutenvolumen von ca. 1201) zu beachten: die für die Humanmedizin konzipierten Geräte und Anschlüsse dürfen nicht durch zu hohe Widerstände zu Dyspnoe oder Panik führen. Werden die Zuführungen in für Pferdelungen-Volumina konzipierte Atemöffnungen integriert, so wäre eine Atemzugtriggerung der Aerosolproduktion ideal. Kann diese nicht verwirklicht werden, so sind unbedingt Filter im Ausatemkanal zu integrieren, damit das kontinuierlich produzierte Aerosol nicht den zwangsläufig in der Nähe stehenden menschlichen Betreuer belastet. Eine Filterung sollte grundsätzlich erwogen werden, da bislang keine Untersuchungen zur Kontamination der Umgebung mit ausgeatmeten Arzneimitteln vorliegen.

Die auf den ersten Blick viel einfachere und zeitsparende Gabe von Arzneimittelaerosolen mittels MDI ist zunächst aus einem rechtlichen Grund problematisch: beim Pferd handelt es sich in der Europäischen Gemeinschaft grundsätzlich um eine Lebensmittel liefernde Tierart. Die im Sinne des Verbraucherschutzes erlassenen Beschränkungen, was die Verwendung von (Human-)Arzneimitteln bei Schlachttieren angeht, verbieten dem Tierarzt oftmals eine medizinisch optimale Patientenversorgung. Vorweggeschickt sei hier gleichwohl, dass die klinische Wirksamkeit von per MDI an Pferde verabreichte Kortikosteroide und Bronchospasmolytika inzwischen gut belegt ist. Allerdings ist die Optimierung derartiger Systeme auf den Bedarf des Pferdepatienten wünschenswert.

Bislang ist die Verwendung von MDI auch aus medizinischen Gründen nicht unproblematisch, da einem Pferd nicht die in der Humanmedizin geforderte, mit dem Sprühstoß zu koordinierende Einatmung beigebracht werden kann. Ein 2002 in den USA zugelassenes, in die Nüster einzuführendes System $3 \mathrm{M}$ Equine Inhaler $^{\mathrm{TM}}$ ( $3 \mathrm{M}$ und Boehringer Ingelheim/Deutschland) zeigt dem Behandler mittels eines Fähnchens, das bei der Inspiration erscheint, den richtigen Zeitpunkt zum Auslösen des Sprühstoßes an. Führt das Aerosol aber zu einer unangenehmen oder unge- wohnten Empfindung, so wird der Pferdepatient gleichwohl die Glottis reflektorisch schließen. Persönliche Zweifel bestehen seitens der Autorin auch, ob die Mehrzahl der Patienten die mehrfache Applikation in die Nase toleriert. Eine dänische Firma bietet dagegen einen großen Spacer Equine Haler ${ }^{\mathrm{TM}}$ (Equine HealthCare, Kopenhagen/Dänemark) mit einer trompetenförmigen, weichen Erweiterung an, die der Nüster äußerlich aufgesetzt wird. Über ein Ventil zwischen Spacer und Trompete wird sichergestellt, dass das zuvor in den Spacer gegebene Aerosol auch über mehrere Atemzüge inspiriert werden kann, es aber den Spacer während der Ausatmung nicht verlassen kann. Die weiteste Verbreitung dürfte bislang die ebenfalls mit Ventilen ausgestattete Equine Aeromask ${ }^{\mathrm{TM}}$ (Trudell Medical International, London Ontario/Kanada) gefunden haben: sie wird seit Anfang der 90er Jahre auf dem anglo-amerikanischen Markt angeboten und zeichnet sich durch ihre Verwendbarkeit sowohl mit Verneblern am Patienten als auch über einen Spacer mit MDI aus. Weiter finden sich nicht geschützte, selbstgefertigte Masken (mit unsicherem, eventuell recht großem Totraum), mit deren Hilfe Aerosole an Pferde verabreicht werden. Bei jedem Applikationssystem muss in der Pferdepraxis zudem auf einfache Handhabung und gute Reinigungs- und Desinfektionsmöglichkeiten geachtet werden.

Problemzusammenfassung: Nicht zu unterschätzen ist die Empfindlichkeit der Patienten gegenüber Geräuschen: während die Gewöhnung an eine relativ gleichbleibende Lärmkulisse oft gelingt, besteht bei vielen Pferden eine regelrechte Aversion gegenüber zischenden Geräuschen, wie sie von MDIs erzeugt werden. Vor dem Kauf eines Applikationssystems sollte ausprobiert werden, inwieweit der indidviduelle Patient daran gewöhnt werden kann. Grundsätzlich wird auch beim so genannten „Luxus“-Tier Pferd das Kosten-Nutzen-Verhältnis zu beachten sein, insbesondere bei der Therapie chronischer Atemwegserkrankungen. Die Wirksamkeit und Dosierung jedes Arzneimittel-Aerosols müssen bei der Tierart Pferd überprüft werden: hier liegt inzwischen eine Reihe von Dosierungsvorschlägen vor. Ein noch zu lösendes Problem liegt in der möglichen Kontamination der Umgebungsluft mit den hochwirksamen inhalativen Arzneimitteln. Untersuchungen zu einer möglichen Belastung des Pflegepersonals sind dringend erforderlich. 